\title{
Manipulation of the Charge State of Single Au Atoms on Insulating Multilayer Films
}

\author{
W. Steurer, ${ }^{1, *}$ J. Repp, ${ }^{2}$ L. Gross, ${ }^{1}$ I. Scivetti, ${ }^{3}$ M. Persson, ${ }^{3}$ and G. Meyer ${ }^{1}$ \\ ${ }^{1}$ IBM Research-Zurich, 8803 Rüschlikon, Switzerland \\ ${ }^{2}$ Institute of Experimental and Applied Physics, University of Regensburg, 93053 Regensburg, Germany \\ ${ }^{3}$ Surface Science Research Centre and Department of Chemistry, University of Liverpool, Liverpool L69 3BX, United Kingdom
}

(Received 4 July 2014; published 22 January 2015)

\begin{abstract}
We show charge-state manipulation of single $\mathrm{Au}$ adatoms on 2-11 monolayer (ML) thick $\mathrm{NaCl}$ films on $\mathrm{Cu}$ surfaces by attaching or detaching single electrons via the tip of an atomic force microscope (AFM). Tristate charge control (neutral, negatively charged, and positively charged) is achieved. $\mathrm{On} \mathrm{Cu}(100)$ and $\mathrm{Cu}(111)$ supports, charge tristability is achieved independently of the $\mathrm{NaCl}$ layer thickness. In contrast, on $\mathrm{Cu}(311)$, only $\mathrm{Au}$ anions are stable on the thinnest $\mathrm{NaCl}$ films, but neutral and positive charge states become sufficiently long lived on films thicker than 4 ML to allow AFM-based charge-state-manipulation experiments.
\end{abstract}

DOI: 10.1103/PhysRevLett.114.036801

Stabilizing charge states of atoms and molecules, as well as controlled charge-state manipulation, is of great interest in the framework of molecular electronics and is a prerequisite for single-electron-transport experiments on insulating films. It might seem natural to employ bulk insulators and bulklike insulating films [1,2] for these purposes, on which charge states are intrinsically stabilized by the suppression of charge transfer with the substrate. However, controlled manipulation of the charge state of single atoms and molecules is hampered by technical issues on these substrates and has yet to be shown. Perhaps the most severe difficulty is that scanning tunneling microscopy (STM) is ruled out by the insulating nature of the samples. So far, charge-state manipulation has been shown only for single atoms and molecules on bi- and trilayer $\mathrm{NaCl}$ films [3-7], on which STM operation is enabled by a still high tunneling probability through the film and charge states are stabilized by large ionic relaxations of the $\mathrm{NaCl}$ film. In contrast to charge stabilization on bulk insulators, the charge-state-stabilization mechanism on films with nonvanishing tunneling probability depends strongly on the specific properties of adsorbate, film, and substrate [4], and only selected charge states can be stabilized. Despite its important implications for catalysis as well, the transition from films with nonvanishing tunneling probability to bulklike insulating films [8-10] in the context of chargestate manipulation is poorly understood.

Here, we present a detailed study of Au adatoms adsorbed on 2-11 monolayer (ML) thick $\mathrm{NaCl}$ films, denoted as $\mathrm{NaCl}(2-11 \mathrm{ML})$, grown on $\mathrm{Cu}(100), \mathrm{Cu}(111)$, and $\mathrm{Cu}(311)$. For the thickest films, we extend the charge-state control into regions where charges are stabilized by suppressing charge transfer with the substrate. In this film-thickness regime, charge-state control is achieved by attaching or detaching single electrons via the tip of an atomic force microscope (AFM). Owing to the single-electron sensitivity of the AFM
PACS numbers: 73.20.Hb, 68.37.Ef, 73.61.Ng, 82.37.Gk

[11-15], the passage of the electron from the tip to the $\mathrm{Au}$ atom (and vice versa) is observed as a jump in the frequency shift, $\Delta f$, and the direction of the electron path is retrieved from the shift of the local contact potential difference (LCPD). We show that $\mathrm{Au}$ adatoms on $\mathrm{NaCl}$ exhibit charge tristability (neutral, negatively charged, and positively charged) irrespective of the film thickness on all $\mathrm{Cu}$ substrates except $\mathrm{Cu}(311)$. On $\mathrm{Cu}(311)$, which has a lower work function, only $\mathrm{Au}$ anions are stable on few ML thick films because of phonon-mediated electron transfer from the $\mathrm{Cu}(311)$ substrate through the $\mathrm{NaCl}$ film. However, the lifetime of the metastable neutral and positive states increases by 2 orders of magnitude per $\mathrm{NaCl}$ layer due to a reduction of the tunneling probability through the film. On $\mathrm{NaCl}(5 \mathrm{ML}) / \mathrm{Cu}(311)$, their lifetime is of the order of an hour, long enough to permit AFM-based charge-statemanipulation experiments.

$\mathrm{NaCl}$ films with thicknesses from 2-11 ML were prepared on $\mathrm{Cu}(111), \mathrm{Cu}(100)$, and $\mathrm{Cu}(311)$ single crystal substrates following the procedure described in Ref. [16] and the Supplemental Material [17]. Au atoms were deposited onto the sample at $5 \mathrm{~K}$ by sublimation in vacuum. The experiments were performed in a low-temperature STM or AFM based on a qPlus tuning-fork sensor design [19] at $5 \mathrm{~K}$. The $\mathrm{NaCl}$ films on the different $\mathrm{Cu}$ substrates were characterized from topographical STM images, which were typically recorded at large applied sample biases, $V,(V>4 \mathrm{~V})$ and small currents $(<1 \mathrm{pA})$. Wellordered films with monoatomic steps between individual $\mathrm{NaCl}$ terraces were observed on all substrates; a typical STM image before Au deposition, which was recorded on a $\mathrm{NaCl}(6-11 \mathrm{ML}) / \mathrm{Cu}(111)$ film, is shown in Fig. S1. The local film thickness was determined by a fingerprinting technique based on measuring the lifetime of the vacancy state of intentionally created chlorine vacancies by the tunneling current [16]. 
Figure 1 shows the manipulation of the charge state of a single $\mathrm{Au}$ atom adsorbed on a $\mathrm{NaCl}(10 \mathrm{ML}) / \mathrm{Cu}(111)$ film. Manipulation between three different charge states (neutral, negatively charged, and positively charged) could be achieved by single-electron transfer between the tip and the adatom; the adsorption site of each charge state could be determined from AFM imaging. The charge state of the adatom was manipulated step by step in a controlled manner by applying symmetric voltage ramps (from $0 \mathrm{~V}$ to a certain voltage, depending on the desired manipulation and back in $30 \mathrm{~s}$ ). The change in the charge state was revealed from the behavior of the frequency shifts $\Delta f(V)$ as a function of $V$, as shown both schematically [Fig. 1(a)] and in three consecutively performed charge-manipulation events in Figs. 1(b)-1(d). In all cases, a jump is observed in $\Delta f(V)$ during application of the bias ramp. The $\Delta f(V)$ fall onto two distinct parabolas, which are shifted horizontally and vertically with respect to each other. The horizontal shift of the parabola maximum, which is a direct measure of the change of the LCPD, indicates that charge was transferred between the Au adatom and the tip at the time when the jump in $\Delta f(V)$ occurred $[5,20]$. The vertical shift is strongly influenced by the mesoscopic properties of the tip-sample junction (the so-called averaging effects) and extraction of meaningful information - even from the shift direction-is not trivial [21-23]. Figures 1(e)-1(h) show atomically resolved images of the $\mathrm{NaCl}$ film and were obtained by recording $\Delta f$ images in constant-height mode with different height set points on the $\mathrm{NaCl}$ substrate and above the Au adatom. The Au adsorption site was obtained using the imaged sublattice of $\mathrm{Na}$ atoms as a reference [24].

Prior to the charge-manipulation experiment, after STM imaging at a large positive bias, the Au adatom was negatively charged in a site bridging two $\mathrm{Na}$ atoms [Fig. 1(e)]. Application of the bias ramp induced a jump to a less negative $\Delta f$ at $-0.8 \mathrm{~V}$; see Fig. 1(b). The corresponding LCPD shift to smaller bias voltage after the jump indicated that negative charge was transferred from the $\mathrm{Au}$ adatom to the tip. The Au adatom then ended up being neutral in a site on top of a $\mathrm{Cl}$ atom [Fig. 1(f)]. Application of the bias ramp, shown in Fig. 1(c), shifted the LCPD further to a smaller bias voltage. The Au adatom then appeared as a faint protrusion accompanied by two shallow dimples that were separated by more than a lattice spacing [Fig. 1(g)]. This protrusion, which we identify as the position of a positively charged $\mathrm{Au}$ adatom, was slightly off center of the bridge position. No further changes in the $\Delta f$ signal were observed upon ramping the bias to $-4 \mathrm{~V}$. The reversal of the sign of the bias ramp [Fig. 1(d)] restored the initial charge state of the $\mathrm{Au}$ adatom, which was characterized by a significantly greater LCPD value and adsorption in an equivalent but different bridge position than the one in Fig. 1(b). We assume that the fact that the $\mathrm{Au}^{0}$ charge state is not observed on the way back from $\mathrm{Au}^{+}$to $\mathrm{Au}^{-}$is related to a larger voltage threshold for

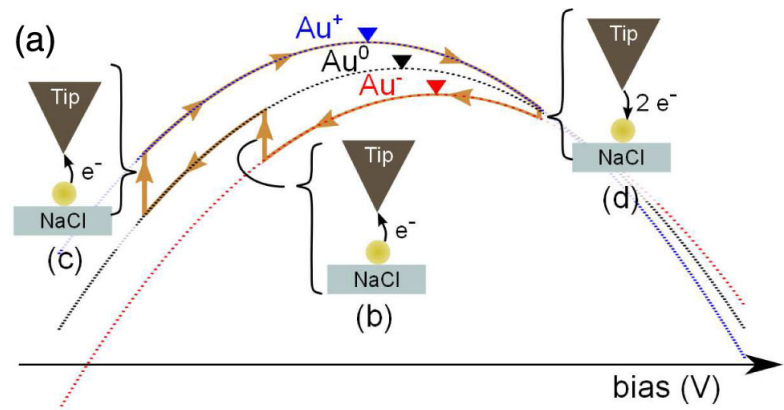

(b)
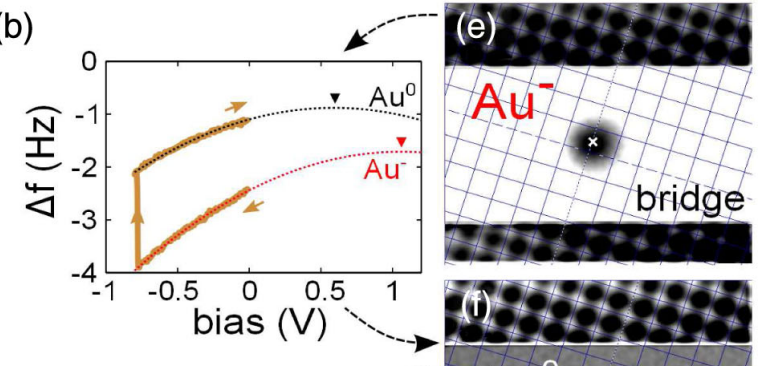

(c)


(d)

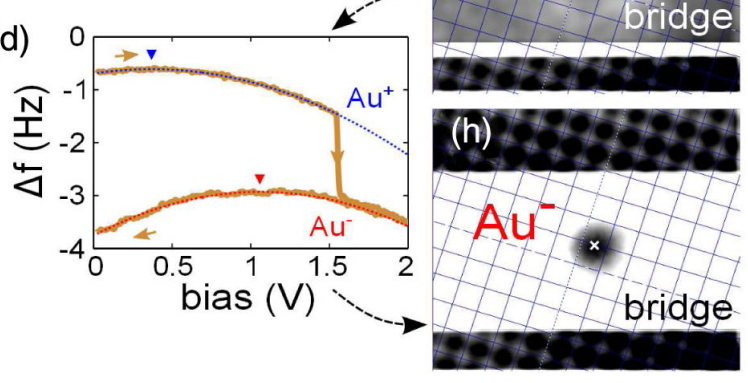

FIG. 1 (color online). Manipulation of the charge state of a $\mathrm{Au}$ adatom on $\mathrm{NaCl}(10 \mathrm{ML}) / \mathrm{Cu}(111)$ by attaching or detaching single electrons via a probe tip. (a) Schematic $\Delta f(V)$ spectrum of a closed charge-switching cycle $\left(\mathrm{Au}^{-} \rightarrow \mathrm{Au}^{0} \rightarrow \mathrm{Au}^{+} \rightarrow \mathrm{Au}^{-}\right)$. The dotted parabolas visualize the change of the LCPD (the triangles) by a change of the charge state. (b)-(d) Experimental manipulation spectra for stepwise manipulation from $\mathrm{Au}^{-} \rightarrow$ $\mathrm{Au}^{0}, \mathrm{Au}^{0} \rightarrow \mathrm{Au}^{+}$, and $\mathrm{Au}^{+} \rightarrow \mathrm{Au}^{-}$, respectively. In each case, the tip was moved above the Au adatom in constant- $\Delta f$ mode and the feedback loop was then interrupted. (e)-(h) Determination of the adsorption position at each stage by constant-height $\Delta f$ images with a different set point on the $\mathrm{NaCl}$ substrate (top and bottom scan lines) and on the Au adatom (middle part). A blue grid is drawn on top of the Na sublattice. Black and white crosses indicate the position of the Au adatom. Imaging conditions are $V=0 \mathrm{~V}, z=13.3$ (8.8) $\AA$ in top and bottom (middle) scan lines, $z=0$ corresponds to a STM set point ( $V=4.2 \mathrm{~V}, I=40 \mathrm{fA}$ ) above $\mathrm{NaCl}$. All images are of the size $50 \times 40 \AA^{2}$. 


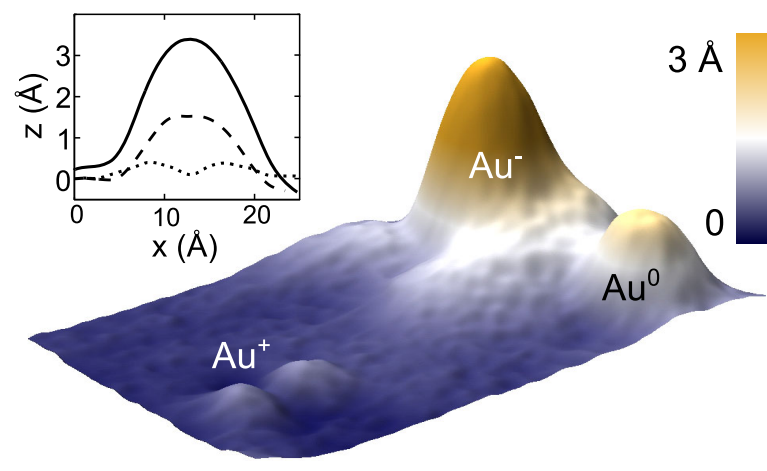

FIG. 2 (color online). Three-dimensional topographical AFM image of three $\mathrm{Au}$ adatoms on a $\mathrm{NaCl}(10 \mathrm{ML}) / \mathrm{Cu}(111)$ film with charge states neutral, positively charged, and negatively charged. Height profiles are shown in the inset figure (solid line: $\mathrm{Au}^{-}$, dashed line: $\mathrm{Au}^{0}$, dotted line: $\mathrm{Au}^{+}$). Imaging details: Const. $\Delta f$ feedback, $\Delta f=-2 \mathrm{~Hz}, V=0 \mathrm{~V}$, image size $60 \times 35 \AA^{2}$.

neutralizing the $\mathrm{Au}^{+}$charge state than the one needed for attaching an electron to a neutral $\mathrm{Au}$ atom. Note that the attachment (detachment) of an electron to (from) the $\mathrm{Au}$ adatom causes its adsorption position to change [see Figs. 1(e)-1(h)] and, therefore, the Au atom will move laterally under the tip in the course of a closed chargeswitching cycle. This motion also affects the vertical and the horizontal shift of the parabolas shown in Figs. 1(b)-1(d). However, the effect on the horizontal shift is small enough that its direction still reflects the change of the charge state (towards being more positive or negative).

Experimental control of the charge state via ramping the bias allowed us to independently set neighboring $\mathrm{Au}$ adatoms to $\mathrm{Au}^{-}, \mathrm{Au}^{0}$, and $\mathrm{Au}^{+}$(see Fig. 2). For example, $\mathrm{Au}^{+}$is set by ramping $V$ to $-2.5 \mathrm{~V}, \mathrm{Au}^{-}$by ramping $V$ to $+2.0 \mathrm{~V}$, and $\mathrm{Au}^{0}$ by first switching to $\mathrm{Au}^{-}$and then ramping $V$ to $-1.0 \mathrm{~V}$. Using constant $\Delta f$ feedback, a topographic AFM image of the ensemble could be obtained without altering the charge state of the individual $\mathrm{Au}$ adatoms during image acquisition. Readout of the charge state without manipulating it is also possible by determining the LCPD; see Fig. 3. In the case of Au adatoms, $\Delta f(V)$ spectra can be measured at short tip-sample distances between -0.6 and $+1.3 \mathrm{~V}$ without changing the charge state [see Figs. 1(b)-1(d)]. However, we found that the LCPD values corresponding to different charge states are often sufficiently distinct to also facilitate charge-state readout at large tip-sample distances for which the tunneling probability through the vacuum gap vanishes [25]. While $\Delta f(V)$ spectra are increasingly influenced by averaging effects at these tip-sample distances, the vanishing tunneling probability allows the measurement over a larger bias range, enabling the LCPD to be determined with a higher precision.

We performed similar charge-manipulation experiments as the one shown in Fig. 1 for all film thicknesses between 2 and $11 \mathrm{ML}$, as well as for $\mathrm{NaCl}$ films grown on the $\mathrm{Cu}(100)$
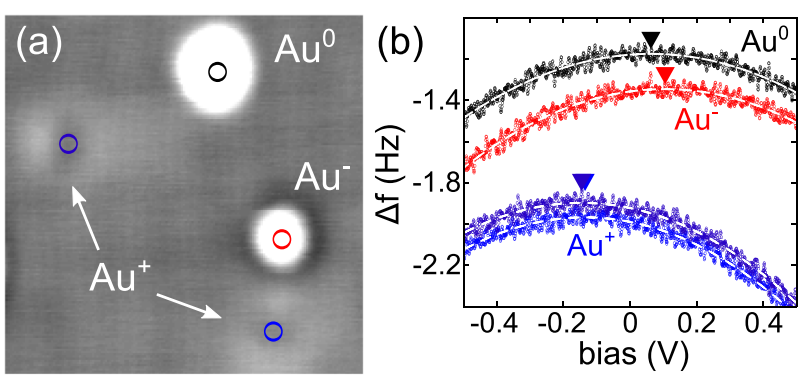

FIG. 3 (color online). Neutral, positively charged, and negatively charged $\mathrm{Au}$ adatoms on a $\mathrm{NaCl}(2 \mathrm{ML}) / \mathrm{Cu}(100)$ film. (a) Constant-current image $(V=0.1 \mathrm{~V}, I=0.45 \mathrm{pA}$, image size $65 \times 65 \AA^{2}$ ). (b) Charge-state readout by recording $\Delta f(V)$ spectra (dots) and determining the LCPD (triangles) from fitted parabolas (dashed lines). The tip was moved above the $\mathrm{Au}$ adatoms in constant-current mode $(V=0.1 \mathrm{~V}, I=0.45 \mathrm{pA})$; the tip position is indicated by circles in (a).

and $\mathrm{Cu}(311)$ substrates. Tristate charge control of $\mathrm{Au}$ adatoms was achieved for all film thicknesses and crystallographic orientations of the copper substrate. However, as will be discussed below, the $\mathrm{Au}^{0}$ and $\mathrm{Au}^{+}$states spontaneously decayed into $\mathrm{Au}^{-}$on thin $\mathrm{NaCl}$ films of only a few monolayers supported by $\mathrm{Cu}(311)$. On all $\mathrm{Cu}$ substrates, and irrespective of the film thickness, the neutral $\mathrm{Au}$ adatom was observed on top of a chlorine atom, and prevalently in the bridge position both for the negative and the positive charge state [26]. Our results thus expand the scope of charge-state control as compared to earlier work [3], where only $\mathrm{Au}^{0}$ and $\mathrm{Au}^{-}$were reported to be stable and where it had been investigated only for $\mathrm{NaCl}$ (2, $3 \mathrm{ML}$ ) films. We note that the $\mathrm{Au}$ cation has similarities to the $\left(\mathrm{AgCl}_{2}\right)^{-}$complex reported in Ref. [4].

In contrast to the $\mathrm{Cu}(100)$ and $\mathrm{Cu}(111)$ substrates, we observed that $\mathrm{Au}$ atoms on $\mathrm{NaCl}(2-4 \mathrm{ML}) / \mathrm{Cu}(311)$ films were negatively charged directly after $\mathrm{Au}$ deposition. Attempts to set the neutral and positive Au charge states via the probe tip failed on these film thicknesses. Only on film thicknesses $\geq 5 \mathrm{ML}$ could we perform the kind of charge-manipulation experiment shown in Fig. 1. For 3 and $4 \mathrm{ML}$ thick $\mathrm{NaCl}$ films, we could measure the lifetime of the $\mathrm{Au}^{0}$ state by applying a sample bias of $V=-1.7 \mathrm{~V}$, which allowed us to detach the electron from the Au anion as soon as it got charged via the $\mathrm{Cu}(311)$ substrate. The charge state of the $\mathrm{Au}$ adatom was detected via the $\Delta f$ signal, and time lapse spectra were recorded. A typical spectrum, recorded on a $\mathrm{NaCl}$ (4 ML) film, is shown in Fig. 4; the white arrow in the STM image [Fig. 4(a)] indicates the position of the tip. The height of the tip above the $\mathrm{Au}$ atom was adjusted to keep perturbations by the electric field of the tip low while maintaining a reasonable tunneling probability across the vacuum gap. At the tip-Au distance of $8 \AA$ chosen, the neutral and negative charge states are well separated in the $\Delta f$ signal, and the lifetime of the $\mathrm{Au}^{-}$charge state is on the order of seconds; see 

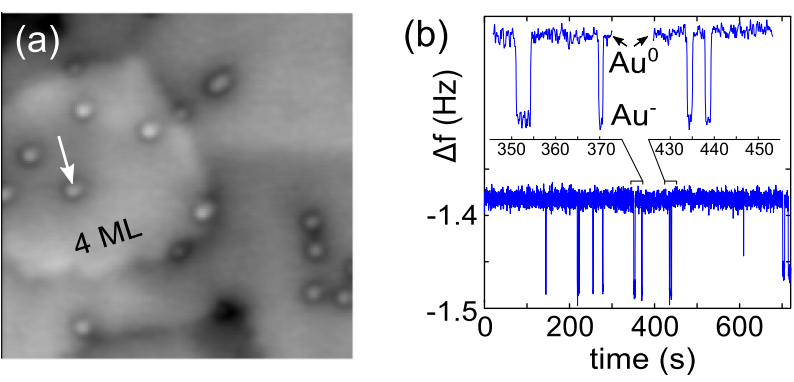

FIG. 4 (color online). Lifetime measurement of the $\mathrm{Au}^{0}$ state on $\mathrm{NaCl}(4 \mathrm{ML}) / \mathrm{Cu}(311)$. (a) STM topography image indicating the position of the tip during data acquisition; the tip was positioned $8 \AA$ above the $\mathrm{Au}$ adatom. Imaging conditions: $V=1.5 \mathrm{~V}, I=20 \mathrm{fA}$, image size $200 \times 200 \AA^{2}$. (b) Time lapse of the $\Delta f$ signal, reflecting the charge state of the Au adatom. A bias of $V=-1.7 \mathrm{~V}$ was applied to transfer the electron from the $\mathrm{Au}$ anion to the tip.

Fig. 4(b). The time between the $\mathrm{Au}^{-}$spikes indicates a lifetime of the $\mathrm{Au}^{0}$ state on the order of $50 \mathrm{~s}$ on the $\mathrm{NaCl}$ (4 ML) film. On $\mathrm{NaCl}$ (3 ML), see the Supplemental Material [17], the lifetime of the $\mathrm{Au}^{0}$ state is at the detection limit imposed by the frequency and amplitude control loops of the AFM force sensor, which are approximately $0.5 \mathrm{~s}$.

In Ref. [4], the key parameters determining the stability of various charge states are discussed within a simple model. Based on this model, we ascribe the spontaneous decay of the $\mathrm{Au}^{0}$ and $\mathrm{Au}^{+}$charge states on $\mathrm{NaCl}$ films grown on $\mathrm{Cu}(311)$ to the significantly reduced work function of $\mathrm{Cu}(311)$ (4.31 eV [27], as compared to $4.59 \mathrm{eV}$ for $\mathrm{Cu}(100)$ and $4.94 \mathrm{eV}$ for $\mathrm{Cu}(111)$ [28]), which shifts the affinity level of $\mathrm{Au}^{0}$ for an unrelaxed ion lattice closer to the Fermi level of the $\mathrm{Cu}(311)$ substrate $(\approx 0.8 \mathrm{eV}$ above $\left.\mathrm{E}_{F}[29]\right)$. Since this affinity level is phonon broadened, phonon-mediated charging of the Au adatom by an electron tunneling from the $\mathrm{Cu}(311)$ substrate through the $\mathrm{NaCl}$ film becomes a possible process. In a simplistic model, the likelihood of this process is given by the product of two probabilities: (i) the probability that the $\mathrm{Au}^{0}$ affinity level is temporarily pushed below the Fermi level by phonons and (ii) the probability for an electron to tunnel through the $\mathrm{NaCl}$ film. The former depends on the alignment of the $\mathrm{Au}^{0}$ affinity level with respect to the Fermi level and its vibrational broadening; the latter probability decreases exponentially with increasing film thickness.

Large ionic relaxations within the $\mathrm{NaCl}$ film are responsible for the fact that the $\mathrm{Au}^{-}$charge state is stable and cannot spontaneously decay to the neutral state on films with a high tunneling probability between substrate and adatom. These relaxations cause a realignment of the electronic levels of the $\mathrm{Au}$ adatom and shift the $\mathrm{Au}^{-}$ ionization level well below the Fermi level of the metal substrate. Our work paves the way to a direct determination of the ionic relaxation energy of charged, adsorbed single atoms, as well as the relaxation energy of single molecules, in the future. The energy at which the attachment or detachment of an electron is observed in Fig. 1 is directly related to the relaxation energy; however, it is influenced by the tip-adatom distance and by vibrational broadening of the affinity or ionization level.

In summary, we have shown charge-state manipulation of single $\mathrm{Au}$ atoms on $\mathrm{NaCl}(2-11 \mathrm{ML})$ films by attaching or detaching single electrons via the tip of an AFM. The fact that the AFM is a very sensitive probe with singleelectron sensitivity and does not rely on charge tunneling allows us to monitor the charge-manipulation process in detail, revealing the instant of the electron passage as well as its direction. Furthermore, our results provide physical insight into the transition from few ML thick films to bulklike insulating films with vanishing tunneling probability in the context of charge-state stability. This prepares the ground for future single-electron-transport experiments where suppressing charge transfer with the substrate is highly desirable because charge-state stabilization becomes independent of material properties, more charge states are accessible, and charge leakage to the substrate is also suppressed for charge transfer between adsorbates.

We thank R. Allenspach for the helpful comments. Financial support from the EU projects ARTIST (Contract No. 243421), PAMS (Contract No. 610446), the ERC Advanced Grant CEMAS, and The Leverhulme Trust (Grant No. F/00 025/AQ) is acknowledged.

*wst@zurich.ibm.com

[1] M. Sterrer, T. Risse, U. Martinez Pozzoni, L. Giordano, M. Heyde, H.-P. Rust, G. Pacchioni, and H.-J. Freund, Phys. Rev. Lett. 98, 096107 (2007).

[2] M. Yulikov, M. Sterrer, M. Heyde, H.-P. Rust, T. Risse, H.-J. Freund, G. Pacchioni, and A. Scagnelli, Phys. Rev. Lett. 96, 146804 (2006).

[3] J. Repp, G. Meyer, F. E. Olsson, and M. Persson, Science 305, 493 (2004).

[4] F. E. Olsson, S. Paavilainen, M. Persson, J. Repp, and G. Meyer, Phys. Rev. Lett. 98, 176803 (2007).

[5] L. Gross, F. Mohn, P. Liljeroth, J. Repp, F. J. Giessibl, and G. Meyer, Science 324, 1428 (2009).

[6] I. Swart, T. Sonnleitner, and J. Repp, Nano Lett. 11, 1580 (2011).

[7] T. Leoni, O. Guillermet, H. Walch, V. Langlais, A. Scheuermann, J. Bonvoisin, and S. Gauthier, Phys. Rev. Lett. 106, 216103 (2011).

[8] P. Frondelius, A. Hellman, K. Honkala, H. Häkkinen, and H. Grönbeck, Phys. Rev. B 78, 085426 (2008).

[9] J. Jung, H. J. Shin, Y. Kim, and M. Kawai, Phys. Rev. B 82, 085413 (2010).

[10] M. Sterrer, T. Risse, M. Heyde, H. P. Rust, and H. J. Freund, Phys. Rev. Lett. 98, 206103 (2007).

[11] L. J. Klein and C. C. Williams, Appl. Phys. Lett. 79, 1828 (2001).

[12] E. Bussmann, N. Zheng, and C. C. Williams, Appl. Phys. Lett. 86, 163109 (2005). 
[13] R. Stomp, Y. Miyahara, S. Schaer, Q. Sun, H. Guo, P. Grutter, S. Studenikin, P. Poole, and A. Sachrajda, Phys. Rev. Lett. 94, 056802 (2005).

[14] J. Zhu, M. Brink, and P. L. McEuen, Appl. Phys. Lett. 87, 242102 (2005).

[15] A. Tekiel, Y. Miyahara, J. M. Topple, and P. Grütter, ACS Nano 7, 4683 (2013).

[16] W. Steurer, L. Gross, and G. Meyer, Appl. Phys. Lett. 104, 231606 (2014).

[17] See Supplemental Material at http://link.aps.org/ supplemental/10.1103/PhysRevLett.114.036801, which includes Ref. [18], for details on sample preparation, conduction-band STM imaging of $\mathrm{NaCl}$, and charge-state stability of $\mathrm{Au}$ adatoms on $\mathrm{NaCl} / \mathrm{Cu}(311)$ films.

[18] J. Repp and G. Meyer, Appl. Phys. A 85, 399 (2006).

[19] F. J. Giessibl, Appl. Phys. Lett. 73, 3956 (1998).

[20] T. König, G. H. Simon, H.-P. Rust, G. Pacchioni, M. Heyde, and H.-J. Freund, J. Am. Chem. Soc. 131, 17544 (2009).

[21] G. H. Enevoldsen, T. Glatzel, M. C. Christensen, J. V. Lauritsen, and F. Besenbacher, Phys. Rev. Lett. 100, 236104 (2008).
[22] F. Bocquet, L. Nony, and C. Loppacher, Phys. Rev. B 83, 035411 (2011).

[23] L. Gross, B. Schuler, F. Mohn, N. Moll, N. Pavliček, W. Steurer, I. Scivetti, K. Kotsis, M. Persson, and G. Meyer, Phys. Rev. B 90, 155455 (2014).

[24] G. Teobaldi, K. Lämmie, T. Trevethan, M. Watkins, A. Schwarz, R. Wiesendanger, and A. L. Shluger, Phys. Rev. Lett. 106, 216102 (2011).

[25] W. Steurer, L. Gross, and G. Meyer (unpublished).

[26] For $\mathrm{Au}^{-}$, the adsorption site reported in Ref. [3] is in apparent contradiction to the one reported here. Detailed investigations revealed that adsorption on top of a chlorine atom on bilayer $\mathrm{NaCl}$ films is a special case of a much richer variability, the details of which will be published elsewhere.

[27] F. E. Olsson, M. Persson, J. Repp, and G. Meyer, Phys. Rev. B 71, 075419 (2005).

[28] P. O. Gartland, S. Berge, and B. J. Slagsvold, Phys. Rev. Lett. 28, 738 (1972).

[29] This was estimated from the $\mathrm{Au}^{0}$ affinity level on $\mathrm{NaCl}(2 \mathrm{ML}) / \mathrm{Cu}(111)$ (see Ref. [3]) and the work function difference between the $\mathrm{Cu}(311)$ and $\mathrm{Cu}(111)$ substrates. 